\title{
Thermal Buckling Analysis for Imperfect Cylindrical Shell with Functionally Graded Material Coating
}

\author{
Zewu Wang ${ }^{\mathrm{a}, \mathrm{b}, *}$, Quanfeng Han ${ }^{\mathrm{a}}$, David H. Nash ${ }^{\mathrm{b}}$, Hongxin You $^{\mathrm{a}}$
}

a) School of Chemical Machinery and Safety, Dalian University of Technology, Dalian 116012, China
b) Department of Mechanical and Aerospace Engineering, University of Strathclyde, Glasgow, G1 1XJ, UK

\begin{abstract}
The shell with functionally graded material (FGM) coating is a novel high temperature resistant structure, which has been increasingly applied in the aerospace, nuclear, turbo machinery and other engineering fields. However, there are some defects for practical structure due to the limitation of manufacturing technique. But relevant theoretical research on the thermal buckling behavior of the imperfect cylindrical shell is rather limited in most open literature. Therefore, this work proposed to establish the theoretical solution of the critical buckling temperature rise of the cylindrical shell with an axisymmetric imperfect and FGM coating based on the Donnell shell theory, Koiter model and Galerkin method. The result shows that the theoretical solution is in exact agreement with the literature. In addition, the influences of the profile of the axisymmetric imperfection, the volume fraction of the ceramic phase and the types of the thermal loading on the thermal buckling behavior of the imperfect cylindrical shell with FGM coating are analyzed comprehensively. The study provides a scientific solution and better understanding for the thermal buckling problem of the imperfect cylindrical shell with FGM coating.
\end{abstract}

Key words: Cylindrical shell; Thermal buckling; FGM coating; Imperfection

\section{Nomenclature}

$\boldsymbol{A}_{s}, \boldsymbol{B}_{s}, \boldsymbol{G}_{m n}, \boldsymbol{H}_{m n} \quad$ Constant coefficients

$\boldsymbol{E} \quad$ Young's modulus

$\boldsymbol{L} \quad$ Length of the shell

M Internal moment per unit length of the shell

$N \quad$ Internal force per unit length of the shell

$\boldsymbol{P}$ Corresponding material properties of the ceramic or metal

$\boldsymbol{R} \quad$ Radius of middle surface of the shell

$\boldsymbol{\Delta} \boldsymbol{T} \quad$ Temperature rise load function

$\Delta \boldsymbol{T}_{\mathbf{0}} \quad$ Environment temperature rise

$\Delta \boldsymbol{T}_{\boldsymbol{c}} \quad$ Critical buckling temperature rise

$V \quad$ Distribution function of the volume fraction of the metal phase or ceramic phase

VT Total volume fraction of the metal phase or ceramic phase in FGM coating

$f \quad$ Internal force function

$\boldsymbol{h}$ Total thickness of the shell

$\boldsymbol{h}_{1} \quad$ Thickness of the FGM coating

$\boldsymbol{h}_{2} \quad$ Thickness of the metal

$\boldsymbol{k} \quad$ Exponent of the volume fraction of the metal phase in FGM coating

$\boldsymbol{m} \quad$ Axial half wave number of the shell

$n$ Circumferential full wave number of the shell

$s \quad$ Half wave number of the imperfection

$\boldsymbol{u} \quad$ Axial deflection of the shell 
$v \quad$ Circumferential deflection of the shell

$\boldsymbol{w} \quad$ Normal deflection of the shell

$\boldsymbol{w}^{*} \quad$ Normal deflection of the initial imperfection of the shell

$z \quad$ Normal coordinate of the shell

$\alpha \quad$ Coefficient of thermal expansion

$\boldsymbol{\kappa} \quad$ Curvature of the shell

$\boldsymbol{\mu}$ Poisson's ratio

$\xi \quad$ Amplitude coefficient of the imperfection

$\sigma \quad$ Normal stress

$\tau \quad$ Shear stress

$\gamma \quad$ Shear strain

$\varepsilon \quad$ Normal strain

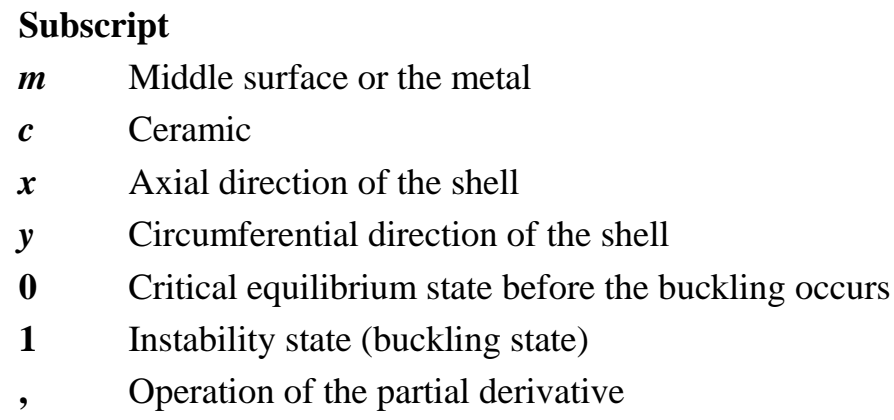

\section{Introduction}

Functionally graded material (FGM), which is usually consisted of the metal phase and ceramic phase in microstructural level, is an innovative heterogeneous composite material [1-3]. Different from normal fibre composites, the components and material properties of the FGM can smoothly and continuously vary along a certain direction according to practical requirements ${ }^{[4-6]}$. Therefore, FGM can avoid thermally or mechanically induced stress gap, which is generally caused by the inconsistency of the material properties at the interface of two different materials for the conventional laminated materials ${ }^{[7,8]}$. Since FGM not only has better mechanical performance, but also exhibits better heat resistance and corrosion resistance than the metal materials in the high temperature environment ${ }^{[9,10]}$, FGMs have been gradually applied in the aerospace, nuclear and other engineering fields in recent years ${ }^{[1-13]}$, particularly for the shells applied in a high temperature or sharp temperature gradient occasion. Although the conception of FGM was proposed in 1980s ${ }^{[14]}$, the cost of pure FGM plate and shell is still rather high at present. Recently, more researchers show the interest in the coat or sandwich FGM plate and shell due to its cost-effective ${ }^{[15-18]}$.

In general, plates and shells are prone to buckle when they are suffered from the mechanical or thermal load, so it is essential for engineers to take into account the stability problem in the design process of these structures. The relevant theories on the buckling behaviour of the pure metal plate and shell were successfully established after many scholars have carried out a great mount of theoretical investigation ${ }^{[19-23]}$. Meanwhile, our previous work ${ }^{[24]}$ also clarified the inconsistency problem of the theoretical critical buckling temperature rise for the pure metallic cylindrical shell in the existing literature ${ }^{[19,20,23]}$ and gave the correct solution. At present, the researchers have paid more attention to the thermal buckling of the composite plate and shell structures. For the rectangular FGM plate with a negative Poisson's ratio, Mansouri and Shariyat ${ }^{[25]}$ investigated the buckling behaviour under a coupled thermo-mechanical load by employing differential orthogonal method. The influences 
of the boundary condition and temperature rise load on the critical buckling load were also discussed in detail. In their recent research, Mansouri and Shariyat ${ }^{[26]}$ derived the theoretical solution of the critical buckling temperature rise for general quadrilateral FGM plate. The influences of the exponent of volume fraction, boundary condition, skew angle of the edge and Poisson's ratio on the buckling behaviour were analysed comprehensively. By utilizing the finite element numerical method, the free vibration and thermal buckling of the FGM plate and cylindrical shell were studied by Kandasamy et al ${ }^{[27]}$. Based on the Donnell shell theory, Wu et al ${ }^{[23]}$ deduced the theoretical solution of the critical buckling temperature rise for pure FGM cylindrical shell. In addition, the mechanical and thermal buckling behaviors of the FGM plate and shell for some special needs, such as the conical and spherical shells, have been studied in recent years ${ }^{[28-30]}$.

The aforementioned a large amount of research work has mainly focused on the perfect plate and shell structures. However, some engineering structures may have local or global initial defective due to the limitation of manufacturing process or operation error. The practical defect could greatly weaken the buckling-resistant ability of the structure because of defect sensitivity. For the cylindrical FGM shell with a local crack, Nasirmanesh and Mohammadi ${ }^{[31]}$ investigated the buckling behavior under the axial tension, axial compression and coupled internal pressure/axial compression conditions using the extended finite element method (XFEM). For the rectangular FGM plate with different types of the local crack, Joshi et al ${ }^{[32]}$ gave the theoretical solution of the critical buckling temperature rise. For the FGM cylindrical shell with global geometric imperfection, the mechanical buckling behavior was studied by Huang and Han by taking into account the influence of temperature on the material properties based on Koiter model ${ }^{[33]}$. Shariat and Eslami ${ }^{[34]}$ derived the theoretical solution of the critical buckling temperature rise for the FGM plate with the global geometric imperfection. In addition, the theoretical study about the nonlinear buckling of the imperfect FGM plates and shells in a thermal environment is also a hot issue ${ }^{[35-37]}$. However, limited work has been reported on the relevant theoretical research of the thermal buckling behaviors of the cylindrical shell with FGM coating and initially geometric imperfect because it not only contains the laminated shell theory, but also involves rather complex material properties of the FGM and geometric structure of the initial imperfect. But, it is necessary to investigate the theoretical solution of the critical buckling temperature rise of the imperfect cylindrical shell with FGM coating for scientific engineering design. Therefore, a first attempt has been made in this work for deriving the general governing equations of the thermal buckling for the imperfect cylindrical shell based on Donnell shell theory and the nonlinear strain-displacement relation of lager deformation. Thereafter, the theoretical critical buckling temperature rise of the cylindrical shell with FGM coating and axisymmetric defect will be established on the basis of Koiter model and Galerkin method by advanced Maple software. The reliability of the theoretical solutions are verified through the solution of perfect cylindrical shell with FGM coating using different theoretical derivation method in our previous work ${ }^{[38]}$, and the influences of the wave number, amplitude of the axisymmetric imperfection, the volume fraction of the ceramic phase and the thermal loading types are discussed in detail. And thus, the developed theoretical solution provides an exact means to design the cylindrical shell with an FGM coating.

\section{Model of the imperfect cylindrical shell with FGM coating}

\subsection{Material model of the cylindrical shell with FGM coating}

As shown in Fig. 1, the original point of the coordinate system is located at the middle surface of the imperfect cylindrical shell in which the inside surface is the metal and the outside surface is the FGM coating, and $x, y$ and $z$ axes are along the axial, circumferential and inner normal directions of the cylindrical shell, respectively. In addition, the axial length of the cylindrical shell is $L$, the radius of the middle surface is $R$, and the thickness of FGM coating and metal are $h_{1}$ and $h_{2}$, respectively.

For the cylindrical shell with FGM coating in Fig. 1, the volume fraction of the metal phase along the normal direction of the shell can be expressed as the following piecewise function 


$$
V_{m}(z)=\left\{\begin{array}{lr}
\left(\frac{2 z+h_{2}}{2 h_{1}}+\frac{1}{2}\right)^{k} & -\frac{h_{1}+h_{2}}{2}<z<\frac{h_{1}-h_{2}}{2} \\
1 & \frac{h_{1}-h_{2}}{2}<z<\frac{h_{1}+h_{2}}{2}
\end{array}\right.
$$

where $V_{m}$ is the volume fraction of the metal phase, $z$ is the normal distance, and $k$ is the exponent of the volume fraction of the metal phase and $k>0$. Meanwhile, the volume fraction of the ceramic phase $V_{c}(z)$ is equal to $1-V_{m}(z)$.

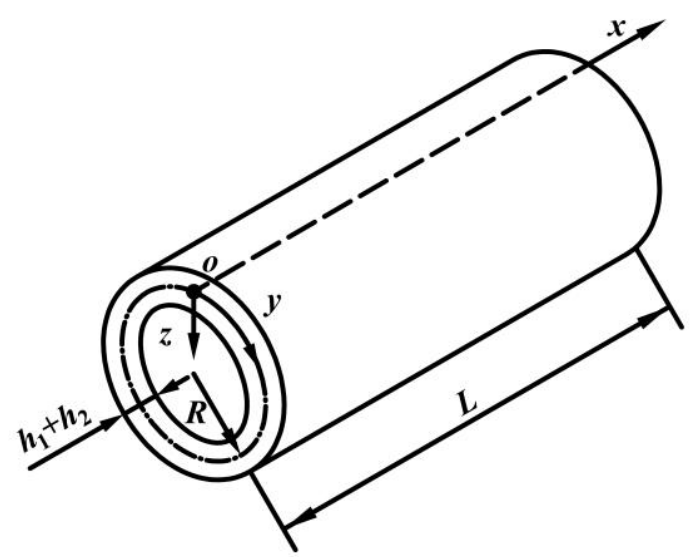

Fig.1 Geometric structure of the cylindrical shell with FGM coating

Based on the linear rule of the mixture that $P(\mathrm{z})=P_{m} V_{m}(z)+P_{c} V_{c}(z)^{[39]}$, the variation function of the material properties with $z$ coordinate are expressed as follows

$$
P(z)=\left\{\begin{array}{lr}
\left(P_{m}-P_{c}\right)\left(\frac{2 z+h_{2}}{2 h_{1}}+\frac{1}{2}\right)^{k}+P_{c} & -\frac{h_{1}+h_{2}}{2}<z<\frac{h_{1}-h_{2}}{2} \\
P_{m} & \frac{h_{1}-h_{2}}{2}<z<\frac{h_{1}+h_{2}}{2}
\end{array}\right.
$$

where $P_{m}$ and $P_{c}$ are the corresponding material properties of the metal and ceramic phase, respectively. Fig 2 shows the variation of the material properties with the different exponent of volume fraction.

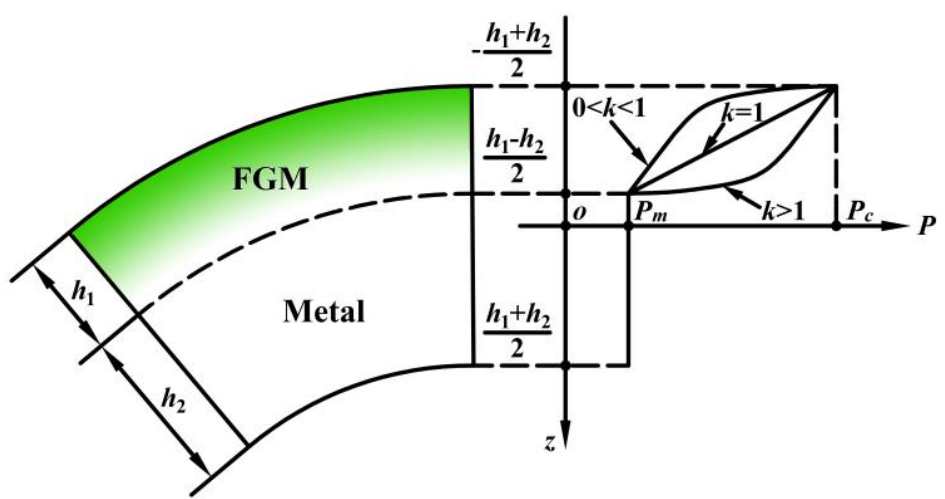

Fig. 2 Schematic of the material property distribution with different $k$

\subsection{Imperfection model of the cylindrical shell with FGM coating}

In the early theoretical and experimental research on the buckling behaviour of the plate and shell structures, some scholars found that there was a big discrepancy between the theoretical and experimental critical buckling loads. For example, the experimental value for the cylindrical shell subjective to the axial compressed load may be only 10 to 20 percentages of the corresponding theoretical value ${ }^{[22]}$. The explanation of this phenomenon is 
also different, but now it is generally acknowledged that the initial imperfection of the structure is the main factor leading to the disagreement between the theoretical and experimental values.

For the plate and shell structures, the imperfections are basically grouped into two categories. One is the geometric imperfection, such as the axisymmetric imperfection caused by the vibration of the cylinder in the high-speed cutting process, as well as the elliptical deviation (Ovality) caused by the large flexibility of the thin-walled cylindrical shell. Another is the material imperfection, such as the delamination due to the thermal-resistant difference among different materials. This work mainly concerns the initial axisymmetric imperfection, and the ovality and delamination will be further investigated in other works.

Since the practical imperfection is rather complicated, it is necessary to simplify the imperfection as an appropriate mathematical model for executing the theoretical analysis. At present, Wan-Donnell model and Koiter model are two major models to describe the axisymmetric geometric imperfection of the cylindrical shell ${ }^{[40]}$. The Wan-Donnell assumes that the initial imperfection is a constantly changing displacement mode, and it doesn't represent a special initial geometric profile. However, the Koiter model introduces the conception of the imperfection coefficient to describe the shape of the initial imperfection. Once the imperfection coefficient is given, the initially geometric profile of the cylindrical shell is determined.

The present work mainly focuses on the axisymmetric imperfection with a given initial geometric shape resulted from the limitation of the manufacturing process. Thus, the Koiter model is chosen to carry out the theoretical analysis since the shape of the initial imperfect is clear.

The Koiter model assumes that the geometric profile of the initial imperfection is axisymmetric ${ }^{[22]}$, and the mathematical expression of the axisymmetric imperfection in the present work can be defined as

$$
w^{*}=\xi h \sin \frac{s \pi x}{L} \quad s=1,2,3, \ldots
$$

where $\xi$ is the amplitude coefficient of the initial imperfection, $h$ is the total wall thickness of the shell, and $h$ $=h_{1}+h_{2} . s$ is the half wave number of the initial imperfection in the $x$ direction. $w^{*}$ is the normal deflection of the initial imperfection of the cylindrical shell. It can be seen from Eq. (3) that the profile of the initial imperfection is mainly dependent on $\xi$ and $s$.

According to Eq. (3), the geometric model of the cylindrical shell is different if the sign of $\xi$ is opposite. Fig. 3 shows two kinds of geometric models of the imperfection cylindrical shell with different $\xi$ while $s=3$. If $\xi$ is larger than 0 , the first point of the maximum deflection should be located at the wave crest in the $x-z$ coordinate system. On the contrary, the first point of the maximum deflection should be at the wave trough if $\xi$ is less than 0 . The geometric model with $\xi>0$ is called as Type-A, and the model with $\xi<0$ is called as Type-B in this work.
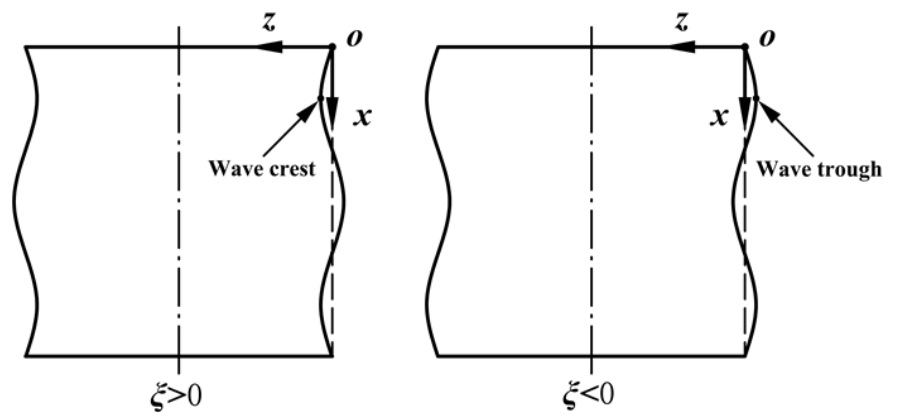

Fig. 3 Schematic of the geometric model of the imperfect cylindrical with different $\xi$

\section{Theoretical derivation of the critical buckling temperature rise}

Similar to literature $[19,23]$, the theoretical analytical solution of the critical buckling temperature rise for the imperfect cylindrical shell with FGM coating is derived on the basis of the following assumptions:

(1) The deformation of the material satisfies the Hooke's law. 
(2) The material properties are temperature independent.

(3) The Poisson's ratios of the metal phase and ceramic phase are equal, which illustrates that $\mu(z)=\mu_{m}=\mu_{\mathrm{c}}=\mu$.

(4) The interface between the metal and coating is perfectly bonded without clearance and sliding.

\subsection{Derivation of the general governing equation of buckling}

For the cylindrical shell, the normal strains in the $x$ and $y$ directions and the shear strain in the $x y$ direction are

$$
\left\{\begin{array}{l}
\varepsilon_{x}=\varepsilon_{x m}+z \kappa_{x} \\
\varepsilon_{y}=\varepsilon_{y m}+z \kappa_{y} \\
\gamma_{x y}=\gamma_{x y m}+2 z \kappa_{x y}
\end{array}\right.
$$

where $\varepsilon_{x m}$ and $\varepsilon_{y m}$ are the normal strain on the middle surface in the $x$ and $y$ directions, respectively, $\gamma_{x y m}$ is the shear strain on the middle surface in the $x y$ directions, $\kappa_{x}, \kappa_{y}$ and $\kappa_{x y}$ are curvatures in the $x, y$ and $x y$ directions, respectively.

Based on the Donnell shell theory, the relations between the deflections and strains on the middle surface are

$$
\begin{array}{lll}
\varepsilon_{x m}=u_{, x}+\frac{1}{2} \kappa_{x}^{2} & \varepsilon_{y m}=v_{, y}+\frac{w}{R}+\frac{1}{2} \kappa_{y}^{2} & \gamma_{x y m}=\left(u_{, y}+v_{, x}\right)+\kappa_{x} \kappa_{y} \\
\kappa_{x}=-w_{, x x} & \kappa_{y}=-w_{, y y} & \kappa_{x y}=-w_{, x y}
\end{array}
$$

where $u, v$ and $w$ are the deflections in the $x, y$ and $z$ directions, respectively, and (,) denotes the operation of partial derivative.

Meanwhile, the thermo-elastic constitutive equations for the cylindrical shell with the FGM coating are

$$
\left\{\begin{array}{l}
\sigma_{x}=\frac{E(z)}{1-\mu^{2}}\left(\varepsilon_{x}+\mu \varepsilon_{y}\right)-\frac{E(z) \alpha(z) \Delta T(z)}{1-\mu} \\
\sigma_{y}=\frac{E(z)}{1-\mu^{2}}\left(\varepsilon_{y}+\mu \varepsilon_{x}\right)-\frac{E(z) \alpha(z) \Delta T(z)}{1-\mu} \\
\tau_{x y}=\frac{E(z)}{2(1+\mu)} \gamma_{x y}
\end{array}\right.
$$

where $\sigma_{x}$ and $\sigma_{y}$ are the normal stresses in the $x$ and $y$ directions, respectively, and $\tau_{x y}$ is the shear stress in the $x y$ direction. $E(z)$ and $\alpha(z)$ are Young's modulus and the coefficient of thermal expansion along the wall thickness, which can be obtained by Eq. (2) . $\mu$ is the Poisson's ratio, and $\Delta T(z)$ is the temperature rise load function.

Since the internal force $N_{i j}$ and internal moment $M_{i j}$ per unit length of the section can be expressed as follows

$$
N_{i j}=\int_{-\left(h_{1}+h_{2}\right) / 2}^{\left(h_{1}+h_{2}\right) / 2} \sigma_{i j} d z \quad M_{i j}=\int_{-\left(h_{1}+h_{2}\right) / 2}^{\left(h_{1}+h_{2}\right) / 2} \sigma_{i j} z d z
$$

Thus, substituting Eqs. (4) and (6) into Eq. (7) gives 


$$
\begin{aligned}
& N_{x}=\frac{\Omega_{1}\left(\varepsilon_{x m}+\mu \varepsilon_{y m}\right)}{1-\mu^{2}}+\frac{\Omega_{2}\left(\kappa_{x}+\mu \kappa_{y}\right)}{1-\mu^{2}}-\frac{\Phi_{1}}{1-\mu} \\
& N_{y}=\frac{\Omega_{1}\left(\varepsilon_{y m}+\mu \varepsilon_{x m}\right)}{1-\mu^{2}}+\frac{\Omega_{2}\left(\kappa_{y}+\mu \kappa_{x}\right)}{1-\mu^{2}}-\frac{\Phi_{1}}{1-\mu} \\
& N_{x y}=\frac{\Omega_{1} \gamma_{x y m}}{2(1+\mu)}+\frac{\Omega_{2} \kappa_{x y}}{1+\mu} \\
& M_{x}=\frac{\Omega_{2}\left(\varepsilon_{x m}+\mu \varepsilon_{y m}\right)}{1-\mu^{2}}+\frac{\Omega_{3}\left(\kappa_{x}+\mu \kappa_{y}\right)}{1-\mu^{2}}-\frac{\Phi_{2}}{1-\mu} \\
& M_{y}=\frac{\Omega_{2}\left(\varepsilon_{y m}+\mu \varepsilon_{x m}\right)}{1-\mu^{2}}+\frac{\Omega_{3}\left(\kappa_{y}+\mu \kappa_{x}\right)}{1-\mu^{2}}-\frac{\Phi_{2}}{1-\mu} \\
& M_{x y}=\frac{\Omega_{2} \gamma_{x y m}}{2(1+\mu)}+\frac{\Omega_{3} \kappa_{x y}}{1+\mu}
\end{aligned}
$$

where

$$
\begin{aligned}
& \Omega_{1}=\int_{-\left(h_{1}+h_{2}\right) / 2}^{\left(h_{1}+h_{2}\right) / 2} E(z) d z=\frac{\left(h_{1}+h_{2}+k h_{2}\right) E_{m}+k E_{c} h_{1}}{k+1} \\
& \Omega_{2}=\int_{-\left(h_{1}+h_{2}\right) / 2}^{\left(h_{1}+h_{2}\right) / 2} z E(z) d z=\frac{\left(E_{m} h_{1}+2 E_{m} h_{2}+k E_{m} h_{2}-E_{c} h_{1}-2 E_{c} h_{2}-k E_{c} h_{2}\right) k h_{1}}{2(k+1)(k+2)} \\
& \Omega_{3}=\int_{-\left(h_{1}+h_{2}\right) / 2}^{\left(h_{h}+h_{2}\right) / 2} z^{2} E(z) d z=\frac{J_{1} h_{1}^{3}+J_{2} h_{1}^{2} h_{2}+J_{3} h_{1} h_{2}^{2}+J_{4} h_{2}^{3}}{12(k+1)(k+2)(k+3)} \\
& \Phi_{1}=\int_{-\left(h_{1}+h_{2}\right) / 2}^{\left(h_{1}+h_{2}\right) / 2} E(z) \alpha(z) T(z) d z \\
& \Phi_{2}=\int_{-\left(h_{1}+h_{2}\right) / 2}^{\left(h_{2}+h_{2}\right) / 2} z E(z) \alpha(z) T(z) d z \\
& J_{1}=6 E_{m}+3 E_{m} k+3 E_{m} k^{2}+8 E_{c} k+3 E_{c} k^{2}+E_{c} k^{3} \\
& J_{2}=18 E_{m}+15 E_{m} k+12 E_{m} k^{2}+3 E_{m} k^{3}+18 E_{c} k+6 E_{c} k^{2} \\
& J_{3}=18 E_{m}+15 E_{m} k+3 E_{m} k^{2}+18 E_{c} k+15 E_{c} k^{2}+3 E_{c} k^{3} \\
& J_{4}=\left(k^{3}+6 k^{2}+11 k+6\right) E_{m}
\end{aligned}
$$

Thus, the strain on the middle surface of the shell can be expressed by the internal force with solving Eqs. (8.a), (8.b) and (8.c) as follows

$$
\begin{aligned}
& \varepsilon_{x m}=\frac{N_{x}-\mu N_{y}-\Omega_{2} \kappa_{x}+\Phi_{1}}{\Omega_{1}} \\
& \varepsilon_{y m}=\frac{N_{y}-\mu N_{x}-\Omega_{2} \kappa_{y}+\Phi_{1}}{\Omega_{1}} \\
& \gamma_{x y m}=\frac{2}{\Omega_{1}}\left[(1+\mu) N_{x y}-\Omega_{2} \kappa_{x y}\right]
\end{aligned}
$$

Then substituting Eq. (9) into Eqs. (8.d), (8.e) and (8.f) gives 


$$
\begin{aligned}
& M_{x}=\frac{\Omega_{2}}{\Omega_{1}} N_{x}+\frac{\Omega_{1} \Omega_{3}-\Omega_{2}^{2}}{\Omega_{1}\left(1-\mu^{2}\right)}\left(\kappa_{x}+\mu \kappa_{y}\right)+\frac{\Omega_{2}}{(1-\mu) \Omega_{1}} \Phi_{1}-\frac{\Phi_{2}}{1-\mu} \\
& M_{y}=\frac{\Omega_{2}}{\Omega_{1}} N_{y}+\frac{\Omega_{1} \Omega_{3}-\Omega_{2}^{2}}{\Omega_{1}\left(1-\mu^{2}\right)}\left(\kappa_{y}+\mu \kappa_{x}\right)+\frac{\Omega_{2}}{(1-\mu) \Omega_{1}} \Phi_{1}-\frac{\Phi_{2}}{1-\mu} \\
& M_{x y}=\frac{\Omega_{2}}{\Omega_{1}} N_{x y}+\frac{\Omega_{1} \Omega_{3}-\Omega_{2}^{2}}{\Omega_{1}(1+\mu)} \kappa_{x y}
\end{aligned}
$$

Meanwhile, the equilibrium equations for the cylindrical shell are

$$
\begin{aligned}
& N_{x, x}+N_{x y, y}=0 \\
& N_{x y, x}+N_{y, y}=0 \\
& M_{x, x x}+2 M_{x y, x y}+M_{y, y y}-N_{y} / R+N_{x} w_{, x x}+2 N_{x y} w_{, x y}+N_{y} w_{, y y}=0
\end{aligned}
$$

For Eqs. (11.a) and (11.b), taking the partial derivative with respect to $x$ and $y$, respectively, afterwards the following equation can be obtained by adding the two equations

$$
N_{x, x x}+2 N_{x y, x y}+N_{y, y y}=0
$$

Then, substituting Eq. (10) into Eq. (11.c) gives

$$
\begin{aligned}
& M_{x, x x}+2 M_{x y, x y}+M_{y, y y}-N_{y} / R+N_{x} w_{, x x}+2 N_{x y} w_{, x y}+N_{y} w_{, y y} \\
= & \frac{\Omega_{2}}{\Omega_{1}}\left(N_{x, x x}+2 N_{x y, x y}+N_{y, y y}\right)+\frac{\Omega_{1} \Omega_{3}-\Omega_{2}^{2}}{\left(1-\mu^{2}\right) \Omega_{1}}\left(\kappa_{y, y y}+\kappa_{x, x x}+2 \kappa_{x y, x y}+\mu \kappa_{x, y y}+\mu \kappa_{y, x x}-2 \mu \kappa_{x y, x y}\right) \\
& +\frac{\Omega_{2}}{(1-\mu) \Omega_{1}}\left(\Phi_{1, y y}+\Phi_{1, x x}\right)-\frac{1}{(1-\mu)}\left(\Phi_{2, x x}+\Phi_{2, y y}\right)-\frac{N_{y}}{R}+N_{x} w_{, x x}+2 N_{x y} w_{, x y}+N_{y} w_{, y y}
\end{aligned}
$$

Thus, substituting Eqs. (5) and (12) into Eq. (13) gives

$$
\begin{aligned}
\frac{\Omega_{1} \Omega_{3}-\Omega_{2}^{2}}{\left(1-\mu^{2}\right) \Omega_{1}} \nabla^{4} w+\frac{N_{y}}{R} & -N_{x} w_{, x x}-2 N_{x y} w_{, x y}-N_{y} w_{, y y} \\
& -\frac{\Omega_{2}}{(1-\mu) \Omega_{1}}\left(\Phi_{1, x x}+\Phi_{1, y y}\right)+\frac{1}{(1-\mu)}\left(\Phi_{2, x x}+\Phi_{2, y y}\right)=0
\end{aligned}
$$

where $\nabla^{4} w=w_{, x x x}+2 w_{, x x y}+w_{, y y y}$.

For the cylindrical shell with an initial axisymmetric imperfection, the normal deflection of the shell $w$ is replaced by $w+w^{*}$. Correspondingly, according to Eq. (5), the expressions of the strains on the middle surface for the imperfect cylindrical shell become

$$
\begin{aligned}
& \varepsilon_{x m}=u_{, x}+\frac{1}{2}\left[\left(w+w^{*}\right)_{, x}\right]^{2}-\frac{1}{2}\left(w_{, x}^{*}\right)^{2}=u_{, x}+\frac{1}{2} w_{, x}^{2}+w_{, x}^{*} w_{, x} \\
& \varepsilon_{y m}=v_{, y}+\frac{w+w^{*}}{R}+\frac{1}{2}\left(w_{, y}+w_{, y}^{*}\right)^{2}-\frac{w^{*}}{R}-\frac{1}{2}\left(w_{, y}^{*}\right)^{2}=v_{, y}+\frac{w}{R}+\frac{1}{2} w_{, y}^{2}+w_{, y} w_{, y}^{*} \\
& \gamma_{x y m}=u_{, y}+v_{, x}+\left(w+w^{*}\right)_{, x}\left(w+w^{*}\right)_{, y}-w_{, x}^{*} w_{, y}^{*}=u_{, y}+v_{, x}+w_{, x} w_{, y}+w_{, x} w_{, y}^{*}+w_{, x}^{*} w_{, y}
\end{aligned}
$$

It is important to note that the internal moments don't depend on the value of the curvature but only on the amount of change of the curvature ${ }^{[41]}$. Thus, the expression of Eq. (14) for the imperfect cylindrical shell should be

$$
\begin{aligned}
\frac{\Omega_{1} \Omega_{3}-\Omega_{2}^{2}}{\left(1-\mu^{2}\right) \Omega_{1}} \nabla^{4} w+\frac{N_{y}}{R} & -N_{x}\left(w+w^{*}\right)_{, x x}-2 N_{x y}\left(w+w^{*}\right)_{, x y}-N_{y}\left(w+w^{*}\right)_{, y y} \\
& -\frac{\Omega_{2}}{(1-\mu) \Omega_{1}}\left(\Phi_{1, x x}+\Phi_{1, y y}\right)+\frac{1}{(1-\mu)}\left(\Phi_{2, x x}+\Phi_{2, y y}\right)=0
\end{aligned}
$$


In addition, assuming that the temperature gradient is distributed only along the wall thickness of the cylindrical shell, $\Phi_{1, x x}, \Phi_{1, y y}, \Phi_{2, x x}$ and $\Phi_{2, y y}$ should be equal to 0 . Thus, before the thermal buckling occurs, the equilibrium equation of Eq. (15) should be written as

$$
\frac{\Omega_{1} \Omega_{3}-\Omega_{2}^{2}}{\left(1-\mu^{2}\right) \Omega_{1}} \nabla^{4} w_{0}+\frac{N_{y 0}}{R}-N_{x 0}\left(w_{0}+w^{*}\right)_{, x x}-2 N_{x y 0}\left(w_{0}+w^{*}\right)_{, x y}-N_{y 0}\left(w_{0}+w^{*}\right)_{, y y}=0
$$

where $w_{0}$ is the normal deflection of the shell caused by the thermal load before the buckling occurs. Correspondingly, $N_{x 0}, N_{y 0}$ and $N_{x y 0}$ are the internal force in the $x, y$ and $x y$ directions, respectively.

Once the thermal buckling takes place, there is a tiny linear increment for the deflection and internal force. The increment of the normal deflection is defined as $w_{1}$, and the increments of the internal forces are defined as $N_{x 1}, N_{y 1}$ and $N_{x y 1}$, respectively. Then, substituting the initial values and increments of the deflection and internal force into Eq. (17) with eliminating these terms which satisfy the initial equilibrium equation and high order minterms, Eq. (17) becomes

$$
\begin{aligned}
\frac{\Omega_{1} \Omega_{3}-\Omega_{2}^{2}}{\left(1-\mu^{2}\right) \Omega_{1}} \nabla^{4} w_{1} & +\frac{N_{y 1}}{R}-N_{x 0} w_{1, x x}-2 N_{x y 0} w_{1, x y}-N_{y 0} w_{1, y y} \\
& -N_{x 1}\left(w_{0, x x}+w_{, x x}^{*}\right)-2 N_{x y 1}\left(w_{0, x y}+w_{, x y}^{*}\right)-N_{y 1}\left(w_{0, y y}+w_{, y y}^{*}\right)=0
\end{aligned}
$$

Introducing the internal force function $f$ which satisfies the following requirements

$$
N_{x 1}=f_{, y y} \quad N_{y 1}=f_{, x x} \quad N_{x y 1}=-f_{, x y}
$$

Substituting Eq. (19) into Eq. (18) gives

$$
\begin{aligned}
\frac{\Omega_{1} \Omega_{3}-\Omega_{2}^{2}}{\left(1-\mu^{2}\right) \Omega_{1}} \nabla^{4} w_{1} & +\frac{f_{, x x}}{R}-N_{x 0} w_{1, x x}-2 N_{x y 0} w_{1, x y}-N_{y 0} w_{1, y y} \\
& -f_{, y y}\left(w_{0, x x}+w_{, x x}^{*}\right)+2 f_{, x y}\left(w_{0, x y}+w_{, x y}^{*}\right)-f_{, x x}\left(w_{0, y y}+w_{, y y}^{*}\right)=0
\end{aligned}
$$

According to Eq. (15), the increments of the strains on the middle surface which ignore the high order minterms are

$$
\begin{aligned}
& \varepsilon_{x m 1}=u_{1, x}+\left(w_{0, x}+w_{, x}^{*}\right) w_{1, x} \\
& \varepsilon_{y m 1}=v_{1, y}+\frac{w_{1}}{R}+\left(w_{0, y}+w_{, y}^{*}\right) w_{1, y} \\
& \gamma_{x y m 1}=u_{1, y}+v_{1, x}+w_{1, x}\left(w_{0, y}+w_{, y}^{*}\right)+w_{1, y}\left(w_{0, x}+w_{, x}^{*}\right)
\end{aligned}
$$

Based on the geometric compatibility condition, the following equation can be obtained through Eq. (21)

$$
\varepsilon_{x m 1, y y}+\varepsilon_{y m 1, x x}-\gamma_{x y m 1, x y}=\frac{w_{1, x x}}{R}+2\left(w_{0, x y}+w_{, x y}^{*}\right) w_{1, x y}-\left(w_{0, y y}+w_{, y y}^{*}\right) w_{1, x x}-\left(w_{0, x x}+w_{, x x}^{*}\right) w_{1, y y}
$$

In addition, according to Eq. (9), another incremental expression form of the strain on the middle surface is

$$
\begin{aligned}
& \varepsilon_{x m 1}=\frac{N_{x 1}-\mu N_{y 1}-\Omega_{2} \kappa_{x 1}}{\Omega_{1}} \\
& \varepsilon_{y m 1}=\frac{N_{y 1}-\mu N_{x 1}-\Omega_{2} \kappa_{y 1}}{\Omega_{1}} \\
& \gamma_{x y m 1}=\frac{2}{\Omega_{1}}\left[(1+\mu) N_{x y 1}-\Omega_{2} \kappa_{x y 1}\right]
\end{aligned}
$$

Thus, substituting Eqs. (19) and (23) into the left part of Eq. (22) gives

$$
\frac{1}{\Omega_{1}} \nabla^{4} f-\frac{w_{1, x x}}{R}-2\left(w_{0, x y}+w_{, x y}^{*}\right) w_{1, x y}+\left(w_{0, y y}+w_{, y y}^{*}\right) w_{1, x x}+\left(w_{0, x x}+w_{, x x}^{*}\right) w_{1, y y}=0
$$


Eqs. (17), (20) and (24) are the general governing equations of the thermal buckling of the imperfect cylindrical shell, which will be used in the next sections.

\subsection{Solution of critical buckling temperature rise}

Since the mathematical expression of the axisymmetric imperfection is defined as $w^{*}=\xi h \sin (s \pi x / L)$, the approximate solution for $w_{0}$ can be written as

$$
w_{0}=A_{s} \sin \frac{s \pi x}{L}+B_{s} \quad s=1,2,3, \ldots
$$

where $A_{s}$ and $B_{s}$ are the constants.

Because the geometric profile is axisymmetric before the buckling occurs, the deflections should satisfy the following conditions ${ }^{[22]}$

$$
u_{0}=u_{0}(x) ; \quad v_{0} \equiv 0 ; \quad w_{0}=w_{0}(x)
$$

Substituting Eqs. (3), (25) and (26) into Eq. (15.b) gives

$$
\varepsilon_{y m 0}=w_{0} / R
$$

According to our previous work ${ }^{[24]}$, the axial internal force $N_{x 0}$ should be $-\int_{-\left(h_{1}+h_{2}\right) / 2}^{\left(h_{1}+h_{2}\right) / 2} E(z) \alpha(z) T(z) d z$, and $N_{x 0}=-\Phi_{1}$. Thus, the axial strain on the middle surface $\varepsilon_{x m 0}$ can be solved by substituting $N_{x 0}=-\Phi_{1}$ into Eq. (8.a) as follows

$$
\varepsilon_{x m 0}=\frac{-\Omega_{1} \mu \varepsilon_{y m 0}-\left(\kappa_{x 0}+\mu \kappa_{y 0}\right) \Omega_{2}+\left(\mu^{2}+\mu\right) \Phi_{1}}{\Omega_{1}}
$$

Then, substituting Eq. (28) into Eq. (8.b) gives

$$
N_{y 0}=\Omega_{1} \varepsilon_{y m 0}+\Omega_{2} \kappa_{y 0}-(1+\mu) \Phi_{1}
$$

Since $w_{0}$ is only the function of the variable $x$, thus $\kappa_{y 0}=w_{0, y y}=0$. Substituting $\kappa_{y 0}=0$ and $\varepsilon_{y m 0}=w_{0} / R$ into Eq. (29), Eq. (29) degenerates to

$$
N_{y 0}=\Omega_{1} w_{0} / R-(1+\mu) \Phi_{1}
$$

Substituting Eqs. (3), (25), (30) into Eq. (16) gives

$$
\begin{aligned}
\frac{\Omega_{1} \Omega_{3}-\Omega_{2}^{2}}{\left(1-\mu^{2}\right) \Omega_{1}} A_{s}\left(\frac{s \pi}{L}\right)^{4} \sin \frac{s \pi x}{L} & +\frac{\Omega_{1}}{R^{2}} A_{s} \sin \frac{s \pi x}{L}-\Phi_{1} A_{s}\left(\frac{s \pi}{L}\right)^{2} \sin \frac{s \pi x}{L} \\
& -\Phi_{1} \xi h\left(\frac{s \pi}{L}\right)^{2} \sin \frac{s \pi x}{L}+\frac{\Omega_{1}}{R^{2}} B_{s}-\frac{(1+\mu) \Phi_{1}}{R}=0
\end{aligned}
$$

Since Eq. (31) is always established before the thermal buckling occurs, the constants $A_{s}$ and $B_{s}$ can be solved through Eq. (31) as follows

$$
A_{s}=\frac{\Phi_{1} \xi h\left(\frac{s \pi}{L}\right)^{2}}{\frac{\Omega_{1} \Omega_{3}-\Omega_{2}^{2}}{\left(1-\mu^{2}\right) \Omega_{1}}\left(\frac{s \pi}{L}\right)^{4}-\Phi_{1}\left(\frac{s \pi}{L}\right)^{2}+\frac{\Omega_{1}}{R^{2}}} \quad B_{s}=\frac{R}{\Omega_{1}}(1+\mu) \Phi_{1}
$$

Thus, substituting Eqs. (25) and (32) into (30), and the circumferential force $N_{y 0}$ should be

$$
N_{y 0}=\frac{\frac{\Phi_{1} \xi h \Omega_{1}}{R}\left(\frac{s \pi}{L}\right)^{2}}{\frac{\Omega_{1} \Omega_{3}-\Omega_{2}^{2}}{\left(1-\mu^{2}\right) \Omega_{1}}\left(\frac{s \pi}{L}\right)^{4}-\Phi_{1}\left(\frac{s \pi}{L}\right)^{2}+\frac{\Omega_{1}}{R^{2}}} \sin \frac{s \pi x}{L}
$$


Since the temperature gradient is distributed only along the wall thickness, and the cylindrical shell is axisymmetric, the shear force $N_{x y 0}$ should be equal to 0 .

For the cylindrical shell simply supported at two ends, the approximate solution of the deflection $w_{1}$ and stress function $f$ can be taken as the following double trigonometric series ${ }^{[22]}$

$$
w_{1}=\sum_{m=1} \sum_{n=1} G_{m n} \sin \frac{m \pi x}{L} \cos \frac{n y}{R} \quad f=\sum_{m=1} \sum_{n=1} H_{m n} \sin \frac{m \pi x}{L} \cos \frac{n y}{R}
$$

where $G_{m n}$ and $H_{m n}$ are the constants, $m$ and $n$ are the axial half wave number and the circumferential full wave number, respectively.

Substituting $N_{x y 0}=0$, Eqs. (3), (25), (30) and (34) into Eqs. (20) and (24) gives

$$
\begin{aligned}
& \frac{\Omega_{1} \Omega_{3}-\Omega_{2}^{2}}{\left(1-\mu^{2}\right) \Omega_{1}} \sum_{m=1} \sum_{n=1}\left[\left(\frac{m \pi}{L}\right)^{2}+\left(\frac{n}{R}\right)^{2}\right]^{2} G_{m n} \sin \frac{m \pi x}{L} \cos \frac{n y}{R}-\frac{1}{R} \sum_{m=1} \sum_{n=1}\left(\frac{m \pi}{L}\right)^{2} H_{m n} \sin \frac{m \pi x}{L} \cos \frac{n y}{R} \\
& \quad-\Phi_{1} \sum_{m=1} \sum_{n=1}\left(\frac{m \pi}{L}\right)^{2} G_{m n} \sin \frac{m \pi x}{L} \cos \frac{n y}{R}+A_{s} \frac{\Omega_{1}}{R} \sin \frac{s \pi x}{L} \sum_{m=1} \sum_{n=1}\left(\frac{n}{R}\right)^{2} G_{m n} \sin \frac{m \pi x}{L} \cos \frac{n y}{R} \\
& \quad-A_{s}\left(\frac{s \pi}{L}\right)^{2} \sin \frac{s \pi x}{L} \sum_{m=1} \sum_{n=1}\left(\frac{n}{R}\right)^{2} H_{m n} \sin \frac{m \pi x}{L} \cos \frac{n y}{R}-\xi h\left(\frac{s \pi}{L}\right)^{2} \sin \frac{s \pi x}{L} \sum_{m=1} \sum_{n=1}\left(\frac{n}{R}\right)^{2} H_{m n} \sin \frac{m \pi x}{L} \cos \frac{n y}{R}=0 \\
& \frac{1}{\Omega_{1}} \sum_{m=1} \sum_{n=1}\left[\left(\frac{m \pi}{L}\right)^{2}+\left(\frac{n}{R}\right)^{2}\right]^{2} H_{m n} \sin \frac{m \pi x}{L} \cos \frac{n y}{R}+\frac{1}{R} \sum_{m=1} \sum_{n=1}\left(\frac{m \pi}{L}\right)^{2} G_{m n} \sin \frac{m \pi x}{L} \cos \frac{n y}{R} \\
& \quad+A_{s}\left(\frac{s \pi}{L}\right)^{2} \sin \frac{s \pi x}{L} \sum_{m=1} \sum_{n=1}\left(\frac{n}{R}\right)^{2} G_{m n} \sin \frac{m \pi x}{L} \cos \frac{n y}{R}+\xi h\left(\frac{s \pi}{L}\right)^{2} \sin \frac{s \pi x}{L} \sum_{m=1} \sum_{n=1}\left(\frac{n}{R}\right)^{2} G_{m n} \sin \frac{m \pi x}{L} \cos \frac{n y}{R}=0
\end{aligned}
$$

Solving Eq. (35) with the Galerkin method, a set of linear equations in terms of $G_{m n}$ and $H_{m n}$ is obtained as follows

$$
\left\{\begin{array}{l}
\beta_{11} G_{m n}+\beta_{12} H_{m n}=0 \\
\beta_{21} G_{m n}+\beta_{22} H_{m n}=0
\end{array}\right.
$$

where

$$
\begin{aligned}
& \beta_{11}=\frac{\Omega_{1} \Omega_{3}-\Omega_{2}^{2}}{\left(1-\mu^{2}\right) \Omega_{1}}\left[\left(\frac{m \pi}{L}\right)^{2}+\left(\frac{n}{R}\right)^{2}\right]^{2}-\Phi_{1}\left(\frac{m \pi}{L}\right)^{2}+A_{s} \frac{\Omega_{1}}{R}\left(\frac{n}{R}\right)^{2} \frac{4 m^{2}[1-\cos (s \pi)]}{\pi s\left(4 m^{2}-s^{2}\right)} \\
& \beta_{12}=-\frac{1}{R}\left(\frac{m \pi}{L}\right)^{2}-\left(A_{s}+\xi h\right)\left(\frac{n}{R}\right)^{2}\left(\frac{s \pi}{L}\right)^{2} \frac{4 m^{2}[1-\cos (s \pi)]}{\pi s\left(4 m^{2}-s^{2}\right)} \\
& \beta_{21}=\frac{1}{R}\left(\frac{m \pi}{L}\right)^{2}+\left(A_{s}+\xi h\right)\left(\frac{n}{R}\right)^{2}\left(\frac{s \pi}{L}\right)^{2} \frac{4 m^{2}[1-\cos (s \pi)]}{\pi s\left(4 m^{2}-s^{2}\right)} \\
& \beta_{22}=\frac{1}{\Omega_{1}}\left[\left(\frac{m \pi}{L}\right)^{2}+\left(\frac{n}{R}\right)^{2}\right]^{2}
\end{aligned}
$$

In order to ensure that there is non-zero solution for Eq. (36), the determinant of the coefficient matrix of Eq. (36) should be equal to 0 . Thus the coefficient matrix of Eq. (36) satisfies

$$
\left|\begin{array}{cc}
\beta_{11} & \beta_{12} \\
\beta_{21} & \beta_{22}
\end{array}\right|=0
$$

If the temperature load is uniform along the wall thickness of the cylindrical shell, and defining $\Delta T(z)=\Delta T_{0}$, where $\Delta T_{0}$ is the given environmental temperature rise, $\Phi_{1}$ should be 


$$
\begin{aligned}
\Phi_{1} & =\int_{-\left(h_{1}+h_{2}\right) / 2}^{\left(h_{1}+h_{2}\right) / 2} E(z) \alpha(z) T(z) d z \\
& =\frac{\left[\left(E_{m} \alpha_{m}+E_{m} \alpha_{m} k+E_{m} \alpha_{c} k+E_{c} \alpha_{m} k+2 E_{c} \alpha_{c} k^{2}\right) h_{1}+\left(2 k^{2}+3 k+1\right) E_{m} \alpha_{m} h_{2}\right] T_{0}}{(k+1)(2 k+1)}
\end{aligned}
$$

If the temperature load is linear distributed along the wall thickness of the cylindrical shell, and the temperature on the surface of the ceramic is higher, thus the thermal load is defined as $\Delta T(z)=$ $\Delta T_{0}\left[\left(h_{1}+h_{2}\right) / 2-z\right] /\left(h_{1}+h_{2}\right) . \Phi_{1}$ should be

$$
\Phi_{1}=\int_{-\left(h_{1}+h_{2}\right) / 2}^{\left(h_{1}+h_{2}\right) / 2} E(z) \alpha(z) T(z) d z=\frac{\left(Q_{1} h_{1}^{2}+Q_{2} h_{1} h_{2}+Q_{3} h_{2}^{2}\right) T_{0}}{2\left(h_{1}+h_{2}\right)(k+1)(k+2)(2 k+1)}
$$

where

$$
\begin{aligned}
& Q_{1}=2 E_{m} \alpha_{m}+E_{m} \alpha_{m} k+3 E_{m} k \alpha_{c}+3 E_{c} \alpha_{m} k+7 E_{c} \alpha_{c} k^{2}+2 E_{c} \alpha_{c} k^{3} \\
& Q_{2}=4 E_{m} \alpha_{m}+6 E_{m} \alpha_{m} k+2 E_{m} \alpha_{m} k^{2}+4 E_{m} \alpha_{c} k+2 E_{m} \alpha_{c} k^{2}+4 E_{c} \alpha_{m} k+2 E_{c} \alpha_{m} k^{2}+8 E_{c} \alpha_{c} k^{2}+4 E_{c} \alpha_{c} k^{3} \\
& Q_{3}=2 E_{m} \alpha_{m}+7 E_{m} \alpha_{m} k+7 E_{m} \alpha_{m} k^{2}+2 E_{m} \alpha_{m} k^{3}
\end{aligned}
$$

Substituting Eqs. (38), (39) into Eq. (37), the critical buckling temperature rise of the imperfect cylindrical shell under the uniform and linear temperature rise loads can be solved, respectively. However, the mathematical expression of Eq. (37) is rather complex, and it is very difficult to solve Eq. (37) directly. In this work, the value of the critical buckling temperature rise is calculated using advanced Maple code. In addition, the least value of $\Delta T_{0}$, which is also called as the critical buckling temperature rise $\Delta T_{c r}$, can be obtained by substituting different integers of the axial half wave number $m$ and circumferential full wave number $n$ into Eq. (37).

However, it is worth to be noticed that if the half wave number of the axisymmetric imperfection $s$ is an even number, the terms related to the amplitude coefficient of the imperfection $(\xi)$ will vanish in Eq. (37). As a consequence, the corresponding solution of the critical buckling temperature rise can't be solved, which is also the mathematical limitation of the Koiter model. Thus, only the model in which the half wave number of the defect is an odd number will be carried out in the next sections.

\section{Results and discussion}

The material properties of the metal phase (Aluminum) and ceramic phase (Alumina) are first defined as $E_{m}=70 \mathrm{GPa}, \alpha_{m}=23 \times 10^{-6} /{ }^{\circ} \mathrm{C}, E_{c}=380 \mathrm{GPa}, \alpha_{c}=7.4 \times 10^{-6} /{ }^{\circ} \mathrm{C}$ and $\mu_{m}=\mu_{\mathrm{c}}=0.3$, respectively ${ }^{[28]}$.

\subsection{Verification analysis}

If the amplitude coefficient of the initial imperfection $(\xi)$ is equal to 0 , the cylindrical shell in the present work should be equivalent to a perfect cylindrical shell. Correspondingly, the theoretical solution of the critical buckling temperature rise in this work should be equal to that of the perfect cylindrical shell with FGM coating in Ref. [38].

Fig. 4 shows the comparative result between the present work and our previous work ${ }^{[38]}$ with the given parameters $\left(k=1, h_{1}=4 \mathrm{~mm}\right.$ and $\left.R=L\right)$ under the uniform temperature rise load. In the figure, the red solid line with mark "॰" is the solution in Ref. [38], and the blue solid line with mark " $\diamond$ " is the solution of the present work. It can be seen from Fig. 4 that the critical buckling temperature rises $\left(\Delta T_{c r}\right)$ in the present work with $\xi=0$ are in exact agreement with Ref. [38] even though the theoretical derivation method used in this work is quite different from Ref. [38]. The governing equations in Ref. [38] only depend on the increments of deflection $\left(u_{1}, v_{1}\right.$ and $\left.w_{1}\right)$, but the governing equations in the present work is dependent on not only the increment of deflection $\left(w_{1}\right)$, but also the internal force function $(f)$. Thus, the theoretical solution deduced in the present work is verified to be reliable. 


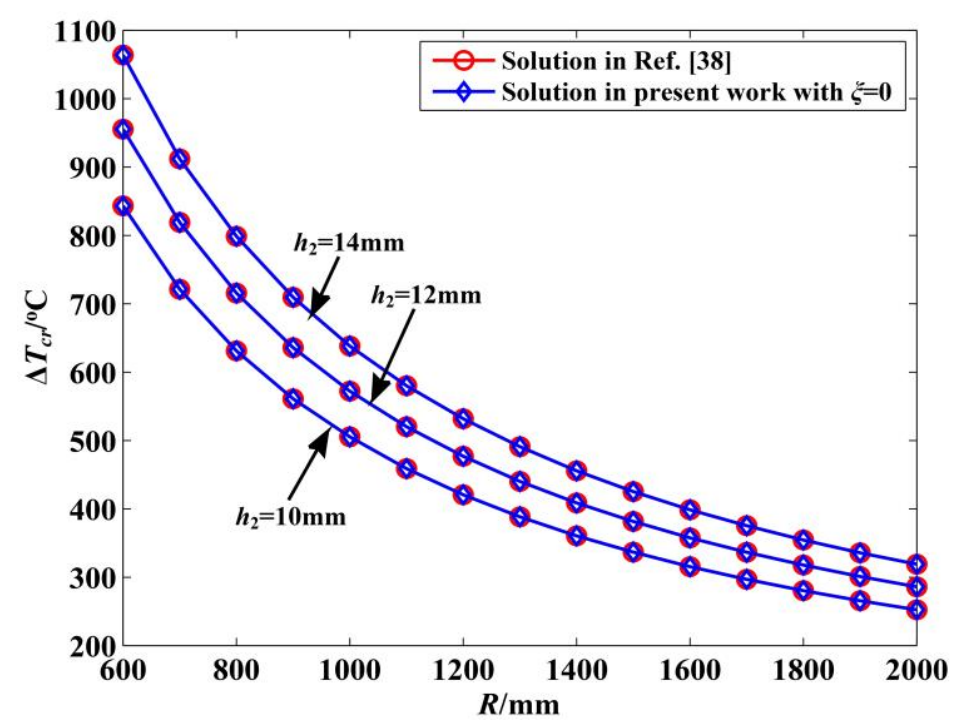

Fig. 4 Comparison among the present work and existing literature

\subsection{Influence analysis of the imperfection structure}

\subsubsection{Influence of the amplitude of the imperfection}

Fig. 5 shows the critical buckling temperature rises of the imperfect cylindrical shell with the given structural parameters $\left(h_{1}=4 \mathrm{~mm}, L=R=1200 \mathrm{~mm}, s=3\right.$ and $\left.k=0.5\right)$. In Fig. 5, the red solid line with mark "०" is the solution of Type-A, and the blue solid line with mark " $x$ " is the solution of Type-B.

As shown in Fig. 5, the critical buckling temperature rise of the imperfect cylindrical shell with FGM coating is nonlinearly and sharply decreased with the increasing of the amplitude coefficient of the imperfection $(\xi)$ for the two models of Type-A and Type-B. For the model of Type-B with $h_{2}=8 \mathrm{~mm}$ in Fig. 5, the critical buckling temperature rise of the imperfect cylindrical shell is even reduced to $26 \%$ of the value of the corresponding perfect $(\xi=0)$ cylindrical shell with FGM coating when $\xi$ is equal to -1 . Fig. 5 illustrates that the critical buckling temperature rise is very sensitive to the amplitude of the imperfection, which illustrates that the initial imperfect obviously weaken the ability of thermal buckling resistance of the cylindrical shell with FGM coating. In addition, it can be seen from Fig. 5 that the critical buckling temperature rise of Type-A is always larger than that of Type-B when the wave number of the initial imperfection is equal to 3 .

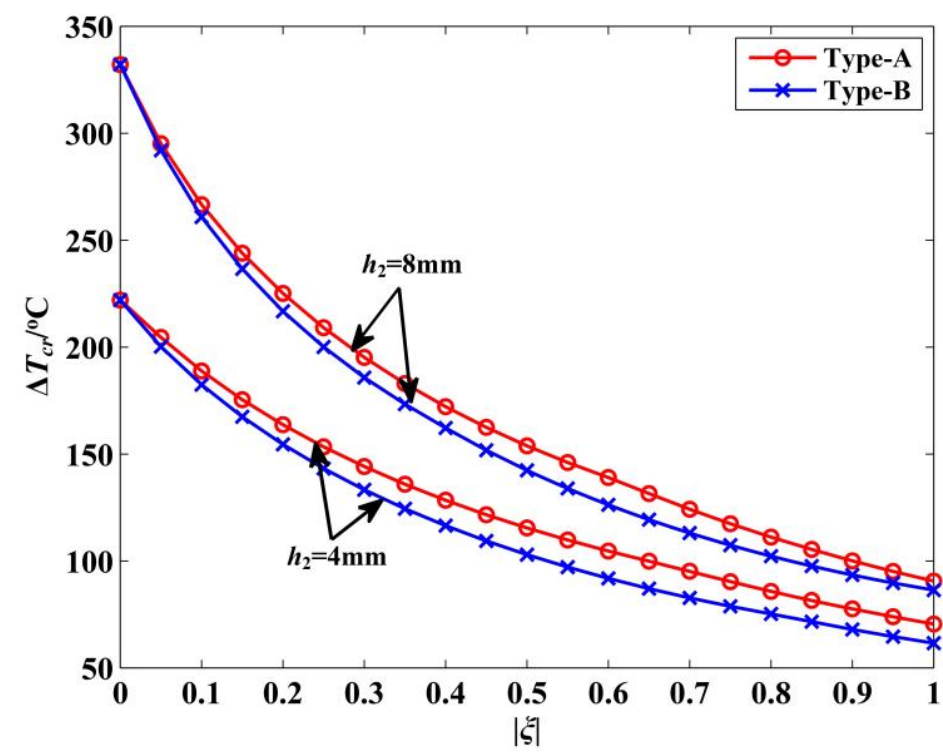

Fig. 5 Influence of $\xi$ on the critical buckling temperature rise 
Table 1 lists the wave number of the cylindrical shell in Fig 5. It can be seen from Table 1 that the axial buckling wave number $(m)$ of the imperfect cylindrical shell is keep constant no matter how $\xi$ changes for the two models of Type-A and Type-B, and the circumferential buckling wave number $(m)$ only decreases by one. It illustrates that the amplitude coefficient of the initial imperfection has little influence on the mode of instability.

Table 1 Influence of $\xi$ on the wave number of buckling

\begin{tabular}{ccccccccc}
\hline & \multicolumn{3}{c}{ Type-A } & \multicolumn{4}{c}{ Type-B } \\
\cline { 2 - 9 }$\xi$ & \multicolumn{3}{c}{$h_{2}=4 \mathrm{~mm}$} & \multicolumn{2}{c}{$h_{2}=8 \mathrm{~mm}$} & \multicolumn{2}{c}{$h_{2}=4 \mathrm{~mm}$} & \multicolumn{3}{c}{$h_{2}=8 \mathrm{~mm}$} \\
\cline { 2 - 9 } & $m$ & $n$ & $m$ & $n$ & $m$ & $n$ & $m$ & $n$ \\
\hline 0 & 6 & 8 & 4 & 8 & 6 & 8 & 4 & 8 \\
0.05 & 1 & 8 & 1 & 7 & 2 & 10 & 2 & 9 \\
0.1 & 1 & 8 & 1 & 7 & 2 & 10 & 2 & 9 \\
0.2 & 1 & 8 & 1 & 7 & 2 & 10 & 2 & 9 \\
0.3 & 1 & 8 & 1 & 7 & 2 & 10 & 2 & 9 \\
0.4 & 1 & 8 & 1 & 7 & 2 & 10 & 2 & 8 \\
0.5 & 1 & 8 & 1 & 7 & 2 & 10 & 2 & 8 \\
0.6 & 1 & 8 & 1 & 7 & 2 & 10 & 2 & 8 \\
0.65 & 1 & 8 & 1 & 6 & 2 & 10 & 2 & 8 \\
0.7 & 1 & 7 & 1 & 6 & 2 & 10 & 2 & 8 \\
0.8 & 1 & 7 & 1 & 6 & 2 & 10 & 2 & 8 \\
0.9 & 1 & 7 & 1 & 6 & 2 & 9 & 2 & 8 \\
1 & 1 & 7 & 1 & 6 & 2 & 9 & 2 & 8 \\
\hline
\end{tabular}

\subsubsection{Influence of the wave number of the imperfection}

Fig. 6 shows the critical buckling temperature rise of the imperfect cylindrical shell under the different wave numbers of the imperfection with the given structural parameters $\left(h_{1}=4 \mathrm{~mm}, L=R=2000 \mathrm{~mm}, k=1\right.$ and $\left.\xi= \pm 0.5\right)$. In Fig. 6, the red solid line with mark "०" is the solution of Type-A, and the blue solid line with mark " $x$ " is the solution of Type-B.

It can be seen from Fig. 6 that the critical buckling temperature rise first decreases, and then increases with the increasing of the wave number $(s)$ of the imperfection when $s$ is less than about 17 for the two models. However, for the Type-A, the critical buckling temperature rise becomes slightly increases when $s$ is larger than about 17. But for the Type-B, it almost keeps constant when $s$ is larger than about 17. In addition, the extreme value of the critical buckle temperature rise for the imperfect cylindrical in Fig. 6 is near to that of the perfect cylindrical shell with the same structural parameters in Fig. 3 for the two models. It illustrates that the critical buckling temperature rise of the imperfect cylindrical shell can be approximately calculated by using the theoretical solution of the perfect cylindrical shell, when the wave number of the initial imperfection is enough large. On the other hand, it also shows that the wave number of the initial imperfection has an obviously negative effect on the critical buckling temperature rise, especially for the case of $s \leq 17$. Thus, it is suggested that the engineer should artificially increase the wave number of the initial imperfection in the manufacturing process to reduce the negative influence of the initial imperfection on the thermal buckling resistance of the cylindrical shell if the manufacturing defect can't be avoided. However, this method is ineffective when the wave number of the imperfection reaches a critical value.

In addition, as above-mentioned in section 4.2.1, the critical buckling temperature rise of Type-A with $s=3$ is always larger than that of Type-B. Fig. 6 also shows the same rule for the model with $s=3$. However, when $s$ is 
larger than 5, the relation of the critical buckling temperature rise between the Type-A and Type-B is contrary as shown in Fig. 6.

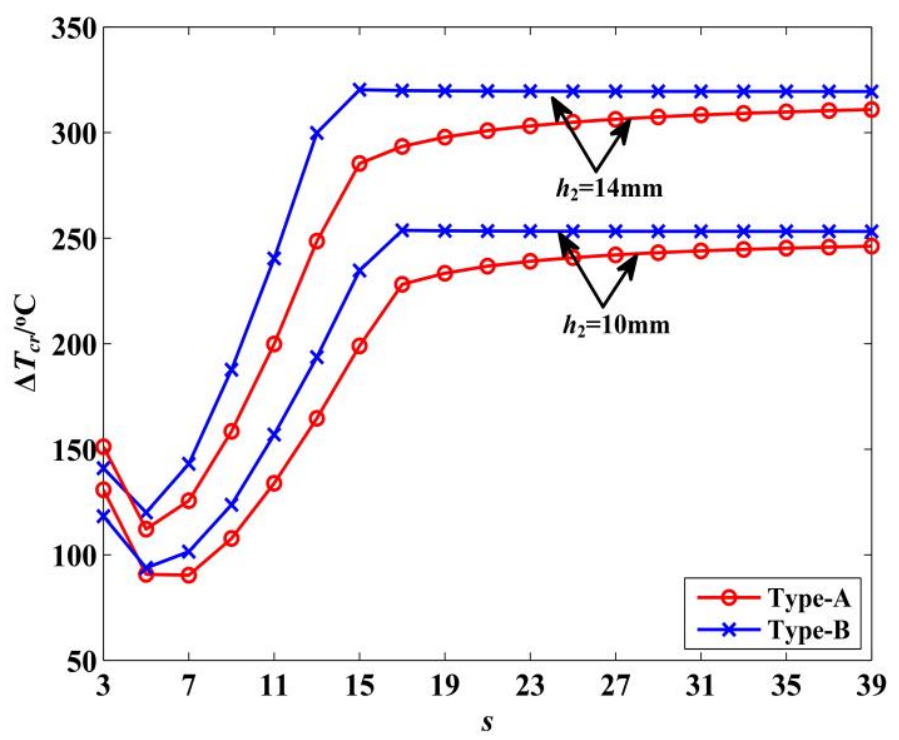

Fig. 6 Influence of $s$ on the critical buckling temperature rise

Table 2 lists the buckling wave numbers of the cylindrical shell in Fig. 6. As shown in Table 2, for the Type-A, the axial buckling wave number $(m)$ is equal to the integer between $s / 2-1$ and $s$ when $s$ is small. However, the axial buckling wave number $(m)$ is equal to the integer between $s / 2$ and $(s+1) / 2$ for the Type-B. In addition, combining the Table 2 and Fig. 6 , it is found that the axial buckling wave number $(m)$ always keeps constant when the wave number of the initial imperfection $(s)$ is greater than 17 changes, and so do the circumferential wave number $(n)$ and the critical buckling temperature rise. Therefore, the conclusion can be drawn that the wave number of the initial imperfection has great influence on the final buckling wave number of the cylindrical shell when $s$ is relatively small, but the effect disappears when $s$ reaches a critical value.

Table 2 Influence of $s$ on the buckling wave number

\begin{tabular}{ccccccccc}
\hline & \multicolumn{4}{c}{ Type-A } & \multicolumn{5}{c}{ Type-B } \\
\cline { 2 - 8 }$s$ & $h_{2}=10 \mathrm{~mm}$ & $h_{2}=14 \mathrm{~mm}$ & \multicolumn{2}{c}{$h_{2}=10 \mathrm{~mm}$} & \multicolumn{2}{c}{$h_{2}=14 \mathrm{~mm}$} \\
\cline { 2 - 8 } & $m$ & $n$ & $m$ & $n$ & $m$ & $n$ & $m$ & $n$ \\
\hline 3 & 1 & 7 & 1 & 7 & 2 & 10 & 2 & 9 \\
5 & 2 & 9 & 2 & 8 & 3 & 10 & 3 & 9 \\
7 & 3 & 10 & 3 & 8 & 4 & 11 & 4 & 9 \\
9 & 4 & 10 & 4 & 8 & 5 & 10 & 5 & 8 \\
11 & 5 & 10 & 5 & 8 & 6 & 10 & 6 & 8 \\
13 & 6 & 9 & 6 & 8 & 7 & 9 & 7 & 7 \\
15 & 7 & 9 & 4 & 9 & 8 & 8 & 6 & 1 \\
17 & 5 & 10 & 3 & 9 & 7 & 1 & 6 & 1 \\
19 & 4 & 11 & 3 & 9 & 7 & 1 & 6 & 1 \\
23 & 4 & 10 & 3 & 9 & 7 & 1 & 6 & 1 \\
27 & 4 & 10 & 3 & 9 & 7 & 1 & 6 & 1 \\
31 & 4 & 10 & 3 & 9 & 7 & 1 & 6 & 1 \\
35 & 4 & 10 & 3 & 9 & 7 & 1 & 6 & 1 \\
39 & 4 & 10 & 3 & 9 & 7 & 1 & 6 & 1 \\
\hline
\end{tabular}




\subsection{Influence of the exponent of volume fraction}

Fig. 7 shows the influence of the exponent of volume fraction $k$ on the critical buckling temperature rise of the imperfect cylindrical shell with the given geometric parameters $\left(h_{1}=h_{2}=4 \mathrm{~mm}, L=R=2000 \mathrm{~mm}\right.$ and $\left.\xi= \pm 1\right)$ under the uniform temperature rise load. In Fig. 7, the blue solid line with mark " $x$ " is the solution of $s=3$, the green solid line with mark " $\boldsymbol{\nabla}$ " is the solution of $s=5$, the red solid line with mark " $\circ$ " is the solution of $s=7$, and the black line with mark " $\diamond$ " is the solution of $s=17$.

Firstly, it can be found from Fig.7 that the critical buckling temperature rise with $\mathrm{s}=17$ is always greater than those with $s=3,5,7$, which is in agreement with the result of section 4.2.2. Thereafter, it can be seen that the curves of the critical buckling temperature rise have nearly vertical tangent at the range that $0.01<k<0.1$. then the growth rate of the critical buckling temperature rise becomes slower and slower when $k$ is larger than 0.1 , and the critical buckling temperature rise even keeps constant when $k>5$. The bigger the $k$ is, the higher the critical buckling temperature rise in Fig.7 is. But the intrinsic relation between the critical buckling temperature rise and $k$ is not yet clear enough.

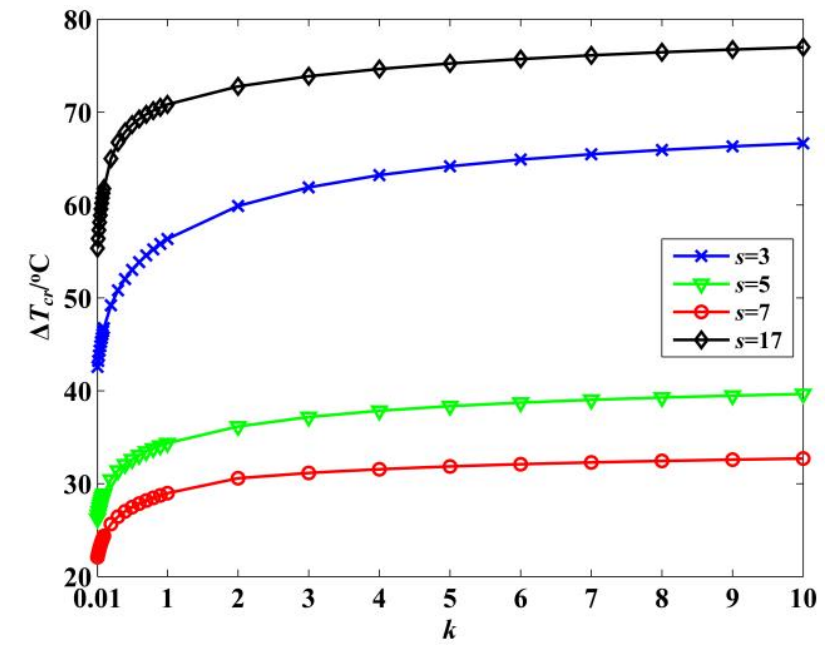

(a) Type-A

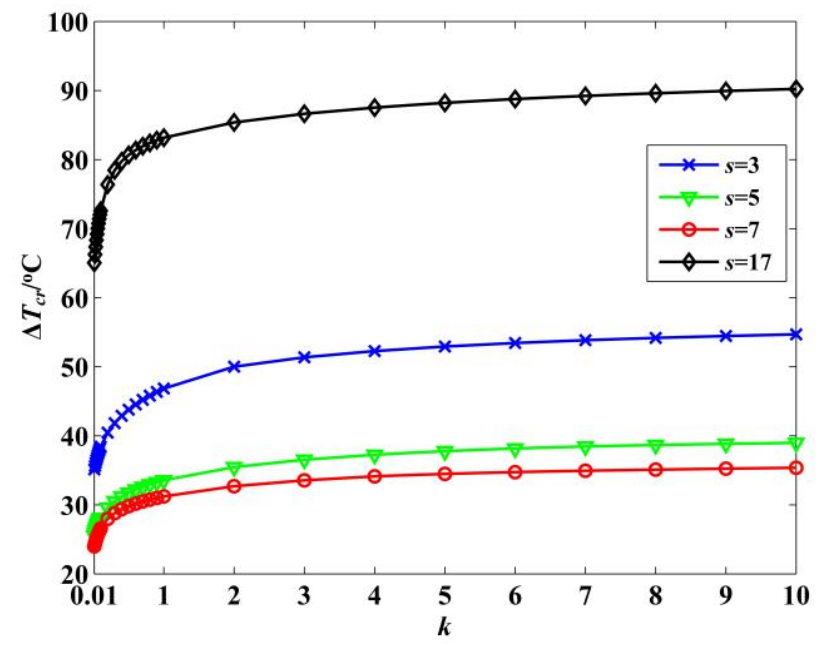

(b) Type-B

Fig. 7 Influence of $k$ on the critical buckling temperature rise

According to Eq. (1), the total volume fraction of the metal and ceramic phase in FGM coating can be obtained as follows

$$
\begin{aligned}
& V T_{m}=\frac{\int_{-\left(h_{1}+h_{2}\right) / 2}^{\left(h_{1}-h_{m}\right) / 2} V_{m}(z) d z}{\int_{-\left(h_{1}+h_{2}\right) / 2}^{\left(h_{1}-h_{2}\right) / 2}\left[V_{m}(z)+V_{c}(z)\right] d z}=\frac{1}{k+1} \\
& V T_{c}=\frac{\int_{-\left(h_{1}+h_{2}\right) / 2}^{\left(h_{1}-h_{2}\right) / 2} V_{c}(z) d z}{\int_{-\left(h_{1}+h_{2}\right) / 2}^{\left(h_{1}-h_{2}\right) / 2}\left[V_{m}(z)+V_{c}(z)\right] d z}=\frac{k}{k+1}
\end{aligned}
$$

It can be seen from Eq. (40) that the total volume fraction of the ceramic phase $\left(V T_{c}\right)$ in FGM coating increases with the increasing of $k$. Note that the FGM coating will be degenerated to the pure metal when $k$ approaches to 0 . Contrarily, if $k$ approaches to infinity, the FGM coating will be degenerated to the pure ceramic.

In order to further study the intrinsic relation between the critical buckling temperature rise and $k$, the horizontal coordinate $k$ in Fig. 7 is replaced by $k /(k+1)$, which is equal to the volume fraction of the metal phase in FGM coating. Hence, the relation between the critical buckling temperature rise and $V T_{c}$ can be gotten as shown in Fig. 8. It can be seen from Fig. 8 that the critical buckling temperature rise rapidly increases with the increasing of $V T_{c}$ when $0<V T_{c}<0.1$. Then, the growth rate gradually slows down, and the critical buckling temperature rise linearly increases with $V T_{c}$ at the range that $0.1<V T_{c}<0.9$. In addition, the critical buckling temperature rise of the 
imperfection cylindrical shell with FGM coating is increased over 39\% when the increasing $V T_{c}$ from 0.01 to 0.91 as shown in Fig. 8.

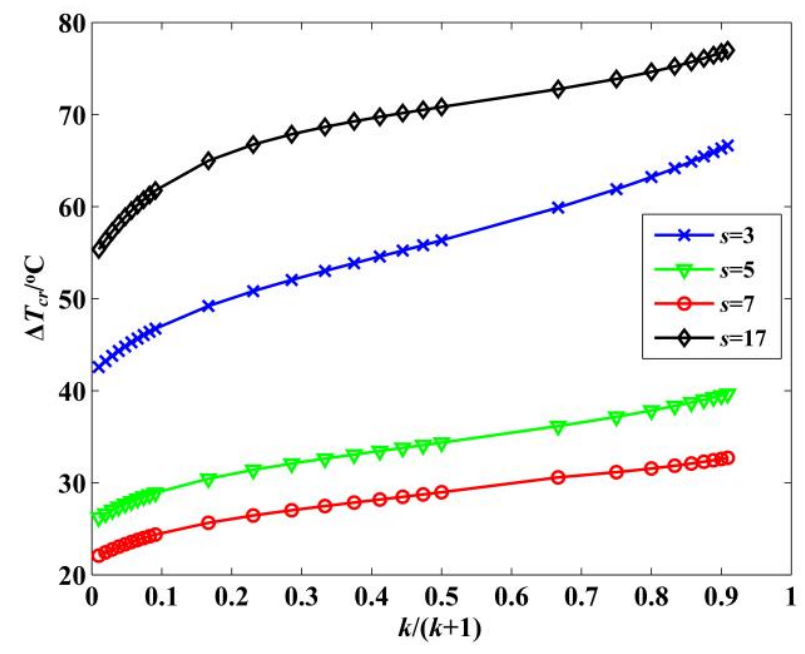

(a) Type-A

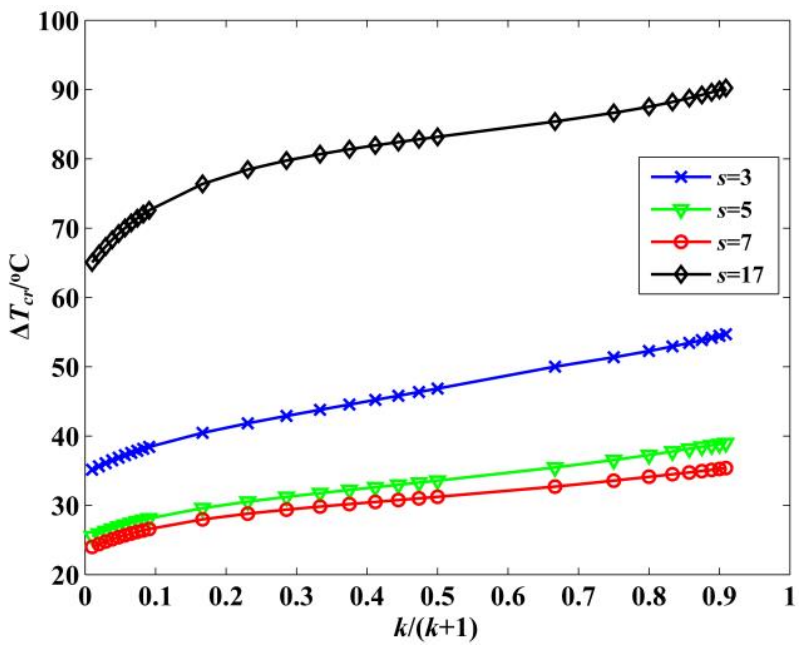

(b) Type-B

Fig. 8 Influence of $V T_{c}$ on the critical buckling temperature rise

Therefore, the increment of the critical buckling temperature rise in Fig. 7 is in fact caused by the increment of the volume fraction of ceramic in FGM coating since the ceramic phase presents larger Young's modulus and smaller coefficient of thermal expansion in comparison with the metal phase. Thus, it is suggested that the designer should increase the volume fraction of the ceramic phase in FGM coating to improve the performance of thermal buckling resistance of the cylindrical shell.

\subsection{Influence of the temperature loading type}

Fig. 9 shows the critical buckling temperature rise of the cylindrical shell with the given parameters ( $h_{1}=2 \mathrm{~mm}, L=R, k=2, r=3$ and $\xi= \pm 0.2$ ) under the uniform and linear temperature rise load conditions, respectively. In Fig. 9, the red solid line with mark "०" is the solution of $h_{2}=10 \mathrm{~mm}$, the green solid line with mark " $\boldsymbol{\nabla}$ " is the solution of $h_{2}=12 \mathrm{~mm}$, and the blue solid line with mark " $x$ " is the solution of $h_{2}=14 \mathrm{~mm}$.

It can be seen from Fig. 9 that the critical buckling temperature rise of the linear temperature rise condition is slightly less than double of the uniform temperature rise load condition, which is similar to the perfect cylindrical shell with FGM coating ${ }^{[38]}$.

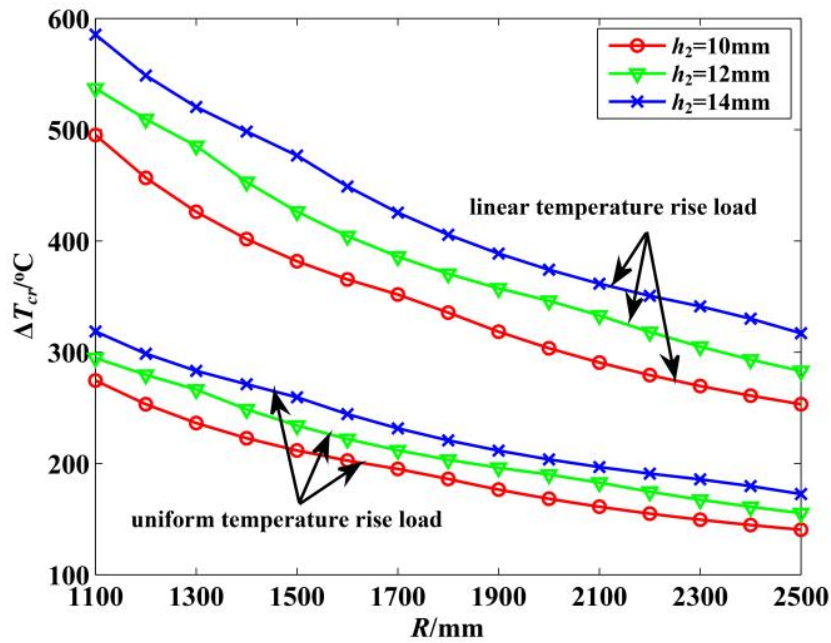

(a) Type-A

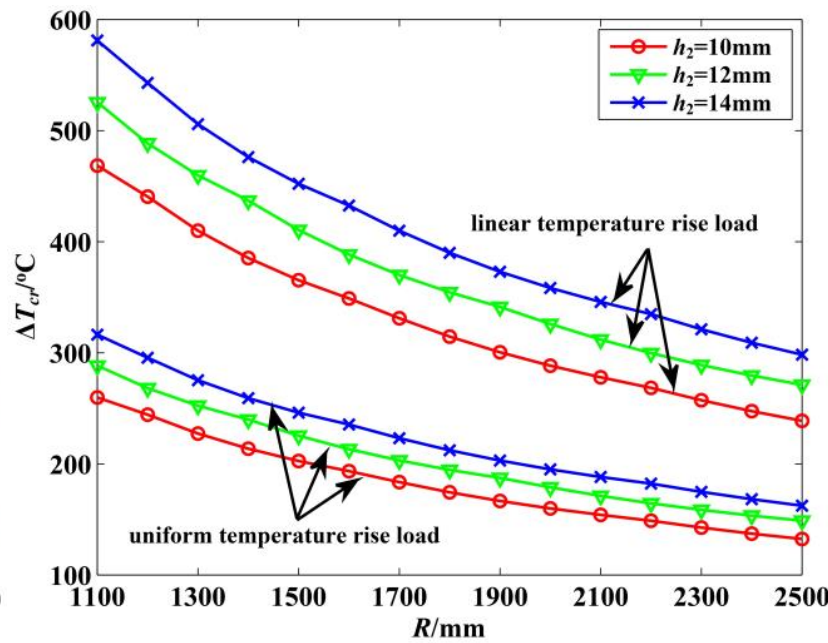

(b) Type-B

Fig. 9 Influence of the thermal load types on the critical buckling temperature rise 


\section{Conclusions}

This work successfully established the theoretical solution of the thermal buckling behavior of the imperfect cylindrical shell with FGM coating based on the Donnell shell theory, Koiter model, Galerkin method and Maple code. Moreover, related factors influencing the performance of thermal buckling resistance were studied comprehensively. The main conclusions are listed as follows:

(1) Based on the different theoretical derivation method, the theoretical solution of the critical buckling temperature rise for the imperfect cylindrical shell with FGM coating in the present work is in exact agreement with the corresponding perfect cylindrical shell, which indicates that the theoretical solution in this work is reliable.

(2) If the axisymmetric imperfection of the cylindrical shell couldn't be completely avoided in the practical manufacturing process, it is suggested that the engineer should artificially fabricate more wave number of the imperfection to relive the negative influence of the imperfection on the critical buckling temperature rise.

(3) When the related geometrical parameters of the cylindrical shell are given, it is suggested that the designer should increase the volume fraction of the ceramic phase in FGM coating for improving the performance of the thermal buckling resistance of the cylindrical shell.

Since the limitation of the Koiter model, only odd wave number of the initial axisymmetric imperfection is investigated in present work, and the influence of the temperature rise on the material properties as well as the other imperfection styles like delamination, are also not taken into consideration. Thus, further investigation on these key points can be studied in the future.

\section{References}

[1] Deniz A, Zerin Z, Karaca Z. Winkler-Pasternak foundation effect on the frequency parameter of FGM truncated conical shells in the framework of shear deformation theory. Composites Part B: Engineering, 2016, 104: 57-70.

[2] Wang Z W, Zhang Q, Xia L Z, et al. Thermomechanical analysis of pressure vessels with functionally graded material coating. Journal of Pressure Vessel Technology, Transactions of the ASME, 2016, 138(011201): 1-10.

[3] Lazar M. A screw dislocation in a functionally graded material using the translation gauge theory of dislocations. International Journal of Solids and Structures, 2011, 48: 1630-1636.

[4] Duc N D, Quang V D, Anh V T T. The nonlinear dynamic and vibration of the S-FGM shallow spherical shells resting on an elastic foundations including temperature effects. International Journal of Mechanical Sciences, 2017, 123: 54-63.

[5] Joodaky A, Joodaky I, Hedayati M, et al. Deflection and stress analysis of thin FGM skew plates on Winkler foundation with various boundary conditions using extended Kantorovich method. Composites Part B: Engineering, 2013, 51: 191-196.

[6] Ansari R, Torabi J, Shojaei M F. Buckling and vibration analysis of embedded functionally graded carbon nanotube-reinforced composite annular sector plates under thermal loading. Composites Part B: Engineering, 2017, 109: 197-213.

[7] Zhao X, Liew K M. Geometrically nonlinear analysis of functionally graded shells. International Journal of Mechanical Sciences, 2009, 51(2): 131-144.

[8] Thai H, Kim S. A review of theories for the modeling and analysis of functionally graded plates and shells. Composite Structures, 2015, 128: 70-86.

[9] Lezgy-Nazargah M. Fully coupled thermo-mechanical analysis of bi-directional FGM beams using NURBS isogeometric finite element approach. Aerospace Science and Technology, 2015, 45: 154-164.

[10] Bich D H, Ninh D G, Thinh T I. Non-linear buckling analysis of FGM toroidal shell segments filled inside 
by an elastic medium under external pressure loads including temperature effects. Composites Part B: Engineering, 2016, 87: 75-91.

[11] Ziane N, Meftah S A, Ruta G, et al. Thermal effects on the instabilities of porous FGM box beams. Engineering Structures, 2017, 134: 150-158.

[12] Naebe M, Shirvanimoghaddam K. Functionally graded materials: A review of fabrication and properties. Applied Materials Today, 2016, 5: 223-245.

[13] Jabbari M, Nejad M Z, Ghannad M. Thermo-elastic analysis of axially functionally graded rotating thick cylindrical pressure vessels with variable thickness under mechanical loading. International Journal of Engineering Science, 2015, 96: 1-18.

[14] Muliana A H. A micromechanical model for predicting thermal properties and thermo-viscoelastic responses of functionally graded materials. International Journal of Solids and Structures, 2009, 46: 1911-1924.

[15] Sofiyev A H, Kuruoglu N. Parametric instability of shear deformable sandwich cylindrical shells containing an FGM core under static and time dependent periodic axial loads. International Journal of Mechanical Sciences, 2015, 101-102: 114-123.

[16] Alibeigloo A. Three dimensional coupled thermo-elasticity solution of sandwich plate with FGM core under thermal shock. Composite Structures, 2017, 177: 96-103.

[17] Do T V, Bui T Q, Yu T T, et al. Role of material combination and new results of mechanical behavior for FG sandwich plates in thermal environment. Journal of Computational Science, 2017, 21: 164-181.

[18] Civalek Ö. Vibration of laminated composite panels and curved plates with different types of FGM composite constituent. Composites Part B: Engineering, 2017, 122: 89-108.

[19] Eslami M R, Ziaii A R, Ghorbanpour A. Thermo-elastic buckling of thin cylindrical shells based on improved stability equations. Journal of Thermal Stresses, 1996, 19(4): 299-315.

[20] Ghorbanpour A. Critical temperature of short cylindrical shells based on improved stability equation. Journal of Applied Sciences, 2002, 2(4): 448-452.

[21] Yang L, Chen Z, Cao G, et al. An analytical formula for elastic-plastic instability of large oil storage tanks. International Journal of Pressure Vessels and Piping, 2013, 101: 72-80.

[22] Brush D O, Almroth B O. Buckling of Bars, Plates, and Shells. New York: McGraw-Hill, 1975.

[23] Wu L, Jiang Z, Liu J. Thermoelastic stability of functionally graded cylindrical shells. Composite Structures, 2005, 70(1): 60-68.

[24] Wang Z, Han Q, Nash D H, et al. Investigation on inconsistency of theoretical solution of thermal buckling critical temperature rise for cylindrical shell. Thin-Walled Structures, 2017, 119: 438-446.

[25] Mansouri M H, Shariyat M. Biaxial thermo-mechanical buckling of orthotropic auxetic FGM plates with temperature and moisture dependent material properties on elastic foundations. Composites Part B: Engineering, 2015, 83: 88-104.

[26] Mansouri M H, Shariyat M. Differential quadrature thermal buckling analysis of general quadrilateral orthotropic auxetic FGM plates on elastic foundations. Thin-Walled Structures, 2017, 112: 194-207.

[27] Kandasamy R, Dimitri R, Tornabene F. Numerical study on the free vibration and thermal buckling behavior of moderately thick functionally graded structures in thermal environments. Composite Structures, 2016, 157: 207-221.

[28] Sofiyev A H, Zerin Z, Kuruoglu N. Thermoelastic buckling of FGM conical shells under non-linear temperature rise in the framework of the shear deformation theory. Composites Part B: Engineering, 2017, 108: 279-290.

[29] Torabi J, Kiani Y, Eslami M R. Linear thermal buckling analysis of truncated hybrid FGM conical shells. Composites Part B: Engineering, 2013, 50: 265-272.

[30] Naj R, Sabzikar Boroujerdy M, Eslami M R. Thermal and mechanical instability of functionally graded truncated conical shells. Thin-Walled Structures, 2008, 46(1): 65-78. 
[31] Nasirmanesh A, Mohammadi S. Eigenvalue buckling analysis of cracked functionally graded cylindrical shells in the framework of the extended finite element method. Composite Structures, 2017, 159: 548-566.

[32] Joshi P V, Jain N K, Ramtekkar G D, et al. Vibration and buckling analysis of partially cracked thin orthotropic rectangular plates in thermal environment. Thin-Walled Structures, 2016, 109: 143-158.

[33] Huang H, Han Q. Buckling of imperfect functionally graded cylindrical shells under axial compression. European Journal of Mechanics - A/Solids, 2008, 27(6): 1026-1036.

[34] Samsam Shariat B A, Eslami M R. Thermal buckling of imperfect functionally graded plates. International Journal of Solids and Structures, 2006, 43(14-15): 4082-4096.

[35] Sahmani S, Aghdam M M. Imperfection sensitivity of the size-dependent post-buckling response of pressurized FGM nanoshells in thermal environments. Archives of Civil and Mechanical Engineering, 2017, 17(3): 623-638.

[36] Duc N D, Thang P T. Nonlinear buckling of imperfect eccentrically stiffened metal-ceramic-metal S-FGM thin circular cylindrical shells with temperature-dependent properties in thermal environments. International Journal of Mechanical Sciences, 2014, 81: 17-25.

[37] Shariyat M, Asgari D. Nonlinear thermal buckling and post-buckling analyses of imperfect variable thickness temperature-dependent bidirectional functionally graded cylindrical shells. International Journal of Pressure Vessels and Piping, 2013, 111-112: 310-320.

[38] Han Q, Wang Z, Nash D H, et al. Thermal buckling analysis of cylindrical shell with functionally graded material coating. Composite Structures, 2017, 181: 171-182.

[39] Duc N D, Thang P T, Dao N T, et al. Nonlinear buckling of higher deformable S-FGM thick circular cylindrical shells with metal-ceramic-metal layers surrounded on elastic foundations in thermal environment. Composite Structures, 2015, 121: 134-141.

[40] Eslami M R, Shahsiah R. Thermal buckling of imperfect cylindrical shells. Journal of Thermal Stresses, 2001.

[41] Timoshenko S P. Theory of elastic stability. McGraw-Hill, 1961. 